\title{
INVESTIGATION OF IMPACT OF TECHNOLOGICAL AND STRUCTURAL PARAMETERS UPON ENERGY INDICATORS OF WORK OF MIXER
}

\author{
Peter Savinyh $^{1}$, Alexey Aleshkin ${ }^{2}$, Nikolai Turbanov ${ }^{1}$, Semjons Ivanovs ${ }^{3}$ \\ ${ }^{1}$ North-East Agricultural Research Institute, Russia; ${ }^{2}$ Vyatka State University, Russia; \\ ${ }^{3}$ Latvia University of Life Sciences and Technologies, Latvia \\ semjons@apollo.lv
}

\begin{abstract}
The main operation in the preparation of balanced feed is high-quality mixing of the components. To date, there are a lot of investigations on the interaction of material particles with the working tools of mixers, yet none of them reflects fully enough the processes that take place in the mixing chamber; therefore, their theoretical studies are topical. The aim of this work is to investigate the impact of the technological and structural parameters upon the energy indicators of the mixer. In carrying out theoretical calculations, methods of analytical mechanics were used. Analysis of the impact of the rotation frequency of the mixer shaft and the material density upon the power consumed for the drive can witness that the rotation frequency of the mixer working tools should not exceed $2.62 \mathrm{rad} \cdot \mathrm{s}^{-1}$, and, before mixing, the components of the mixture should not be heavily disintegrated. Changing the rotation frequency of the mixer shaft and the pitch of the auger turn depending on the value of the consumed power allows to say that the rotation frequency of the mixer working tools in order to reduce energy consumption should not exceed $2.09 \mathrm{rad} \cdot \mathrm{s}^{-1}$, and the pitch of the medium auger should be within the range of 0.4 up to $0.6 \mathrm{~m}$. Investigations of the dependence of power consumption upon the height of the auger belt and its pitch allow a conclusion that, in order to achieve minimum power consumption to drive the mixer working tools, the height of the auger belt should be from 30 to $50 \mathrm{~mm}$, and the pitch of the medium auger belt should be $0.4-0.6 \mathrm{~m}$.
\end{abstract}

Keywords: feed mixing, parameters, energy indicators, frequency.

\section{Introduction}

When keeping farm animals in stationary conditions, the feed base is the most important factor determining the efficiency of animal husbandry. Moreover, not only quantitative indicators are important, but also the fractional composition [1-3].

To date, there are two main directions of preparation of combined animal feed: in large combined feed mills, and directly on the farms.

The main advantage of the feed preparation directly on the farm is that all the peculiarities of the fodder base are immediately taken into account in the region. In designing mixers, a major role is devoted to theoretical studies of the interaction of the mixed components with the surface of the working tools and the mixing chamber.

It is also necessary to consider the interaction of the components themselves with each other, the amount of the material in the mixing chamber, the rotation frequency of the working tools, the particle size, etc.

In order to increase the efficiency of livestock production, it is necessary to use feeds balanced by their nutritional value. The main operation in the preparation of balanced feed is high-quality mixing of the components .

At present, screw-type mixers are prevailing.The auger diameter, the height of the auger, the rotation frequency are of the main design parameters of screw-type feed mixers. The influence of the design parameters and operating modes of feed mixers on energy consumption has not yet been fully studied.

A theoretical study of the impact of structural and technological parameters upon the power consumption when mixing various feed components is a rather complicated task due to the many factors that affect the process [4-6].

Therefore, when making calculations, a necessity arises to use a number of assumptions. To date, there are a lot of investigations on the interaction of material particles with the working tools of mixers [5], yet none of them reflects fully enough the processes that take place in the mixing chamber; therefore, their theoretical studies are topical.

The aim of this work is to investigate the impact of the technological and structural parameters upon the energy indicators of the mixer. 


\section{Materials and methods}

In carrying out theoretical calculations, methods of analytical mechanics were used. For calculations we accept: the specific gravity of the grain mixture $\gamma=0.75 \mathrm{t} \cdot \mathrm{m}^{-3}$; the diameter of the external auger $D_{1}=1 \mathrm{~m}$; the diameter of the medium auger $D_{2}=0.75 \mathrm{~m}$; the diameter of the internal auger $D_{3}=0.4 \mathrm{~m}$; the frequency of rotation of the auger shaft $n=21.5 \mathrm{~min}^{-1}$. The pitch of the external auger $h=0.3 \mathrm{~m}$, the medium auger $h=0.4 \mathrm{~m}$, the internal auger $h=0.24 \mathrm{~m}$. The coefficient of friction of the material when moving along steel is taken to be equal $f=0.4$, the coefficient of internal friction of the material when moving $f_{1}=0.37$. The amount of the reference component is $12 \%$ [7-9].

Let us consider the rotation of a helical surface around its axis in a horizontal mixer of bulk materials, using the theorem about the change of the kinetic moment [10-11]:

$$
I \frac{d \omega}{d t}=M_{V R}+\sum\left(N_{M \varphi} \rho+F_{T R \varphi} \rho\right),
$$

where $I$ - moment of inertia of the mixer shaft together with the helical surface and the material transferred;

$\omega$ - angular velocity of the shaft;

$M_{V R}$ - drive torque applied to the shaft;

$N_{M \varphi}$ - normal reaction of the material, acting upon the elementary area of the helical surface in a projection of its movement, that is, onto the cylindrical axis $\vec{p}$;

$F_{T R \varphi}$ - force of friction of the material applied to the elementary area of the helical surface in a projection onto axis $\vec{p}$;

$\rho$ - radius in the cylindrical coordinates of the elementary surface area, which is the arm of the component forces along direction $\vec{p}$ (Fig. 1).

Fig.1a presents a diagram of the interaction of the helical surface with the material in the mixer, showing the location of the coordinate axes, the direction of the forces, and the position of the elementary site under consideration. Fig.1b depicts an elementary site with the acting forces and the directions of the velocity vectors.
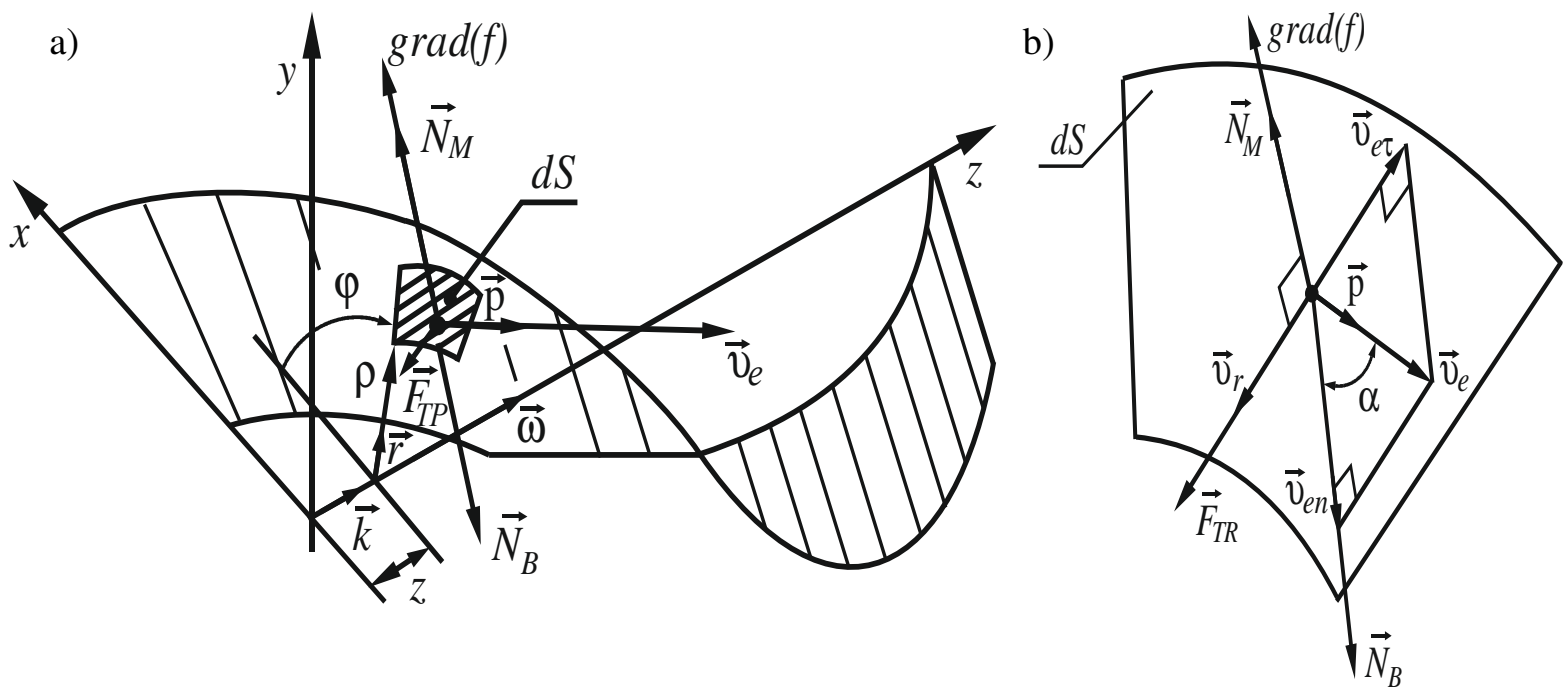

Fig. 1. Scheme for calculation: $a$ - the axis of coordinates and the force; $b$ - the elementary site

When considering the impacts of the material being mixed and the surface of the auger, we make the following assumption. Suppose that the force of friction arises between the elementary volume of the material and the elementary surface area of the auger, and the friction forces between the elementary volumes of the material are small.

The torque required to rotate the mixer shaft at a constant angular velocity $(d \omega / d t=0)$, without regard to friction in the bearings, taking into account expression (1), can be determined by the formula:

$$
M_{V R}=-\sum\left(N_{M \varphi} \rho+F_{T R \varphi} \rho\right) .
$$


The normal reaction $\vec{N}_{M}$ is directed perpendicular to the helical surface (along the gradient). In cylindrical coordinates, the equation of the helical surface is written as follows:

$$
f(\rho, \varphi, z)=z-a \varphi=0 .
$$

By cylindrical vectors $(\vec{r}, \vec{p}, \vec{k})$ the gradient vector to the surface $f(\rho, \varphi, z)$ has the following components:

$$
\operatorname{grad}(f)=\frac{\partial f}{\partial \rho} \vec{r}+\frac{1}{\rho} \frac{\partial f}{\partial \varphi} \vec{p}+\frac{\partial f}{\partial z} \vec{k}
$$

After the partial derivatives are determined, the vector form of the gradient to the surface $f(\rho, \varphi, z)$ will be written:

$$
\operatorname{grad}(f)=-\frac{a}{\rho} \vec{p}+\vec{k}
$$

The gradient modulus will be determined from the expression

$$
|\operatorname{grad}(f)|=\sqrt{\left(\frac{a}{\rho}\right)^{2}+1}
$$

On the axis of the cylindrical coordinates force $\vec{N}_{M}$ is decomposed into components:

$$
\left\{\begin{array}{c}
N_{M \rho}=0 \\
N_{M \varphi}=-\lambda \frac{a}{\rho}, \\
N_{M z}=\lambda
\end{array}\right.
$$

where $\lambda$-indefinite Lagrange multiplier.

According to Coulomb's law, the friction force $\vec{F}_{T R}$ is proportional to the force of normal pressure $\vec{N}_{M}$ with the coefficient $k_{T R}$.

$$
\left|\vec{F}_{T R}\right|=k_{T R}\left|\vec{N}_{M}\right|=k_{T R} \lambda \sqrt{\left(\frac{a}{\rho}\right)^{2}+1} .
$$

The vector of the friction force $\vec{F}_{T R}$ is directed in the direction of relative velocity.

The direction of the friction force $\vec{F}_{T R}$ is opposite to the direction of relative velocity of the movement $\vec{v}_{r}$ along a helical surface.

$$
\vec{F}_{T R}=k_{T R}\left|\vec{N}_{M}\right| \frac{\vec{v}_{r}}{\left|\vec{v}_{r}\right|}
$$

Having determined the projections of the forces entering into equation (2), we write:

$$
N_{M \varphi} \rho+F_{T R \varphi} \rho=-\lambda a-k_{T R} \lambda \rho
$$

Factor $\lambda$ is determined from the correlation

$$
\lambda=\frac{\left|\vec{N}_{M}\right|}{\sqrt{\left(\frac{a}{\rho}\right)^{2}+1}} .
$$

Let us consider the change in the momentum of a certain amount of material interacting with the area $d S$ during time $t$. For calculations we write the theorem in a projection onto the vector gradient to the surface (Fig. 2). Fig. 2 shows a scheme of the interaction of the flow of the accelerated material 1, which is in contact with the elementary area of the screw surface $d S$ and the horizontal layer 2, in the 
form of an oblique cylinder with a base $d S$ and height $h$, the pitch of the screw (the balanced gravity forces and the response reactions are not shown).

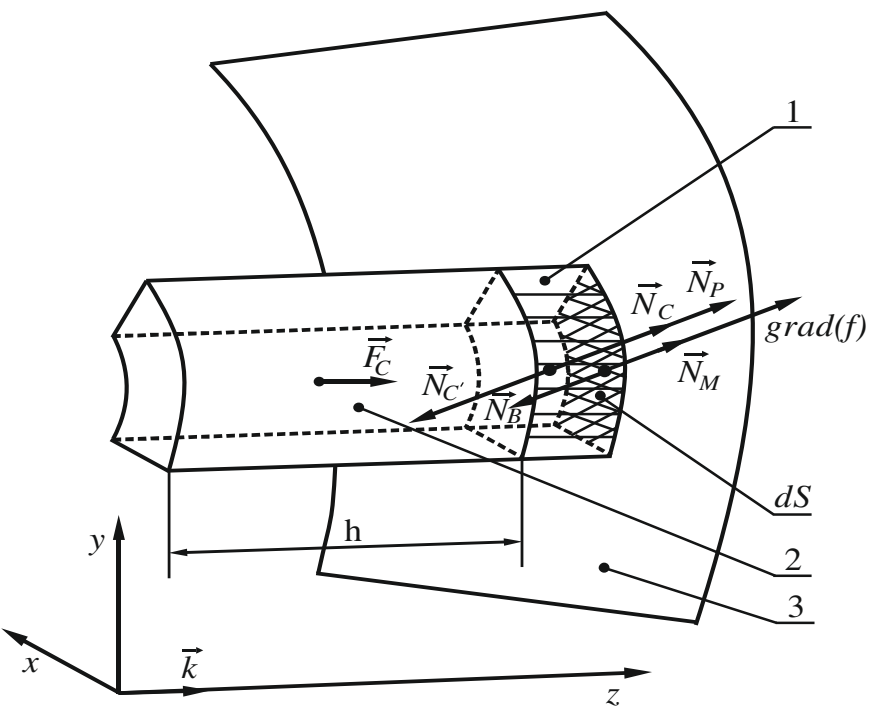

Fig. 2. Scheme of the interaction of the material and the helical surface: 1 - the flow of the accelerated material; 2 - the horizontal layer; 3 - the auger

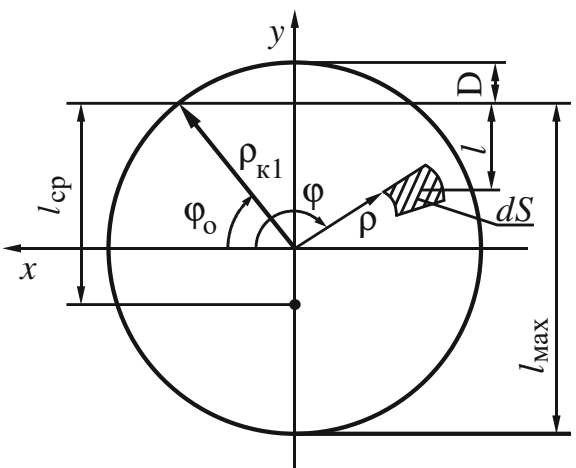

Fig. 3. Scheme of filling the mixer and thickness of the material layer lup to the location of the elementary area of the helical surface $d S$

When compiling the equation, we take into account that, in addition to the helical surface of the auger, forces from the neighbouring fragments are acting upon the fragment of the material studied. We will group these forces in two components: $\vec{N}_{P}$ - the force from the pressure of the overlying layers of the material, and $\vec{N}_{C}$ - the force of normal pressure obtained from the resistance to the horizontal transfer of a part of the material enclosed between the turns of the auger and area $d S$ :

$$
Q_{n}-Q_{n 0}=-\int_{0}^{t} N_{B} d t+\int_{0}^{t} N_{P}+\int_{0}^{t} N_{C}
$$

where $Q_{n}$ - momentum of movement acquired by the material in a projection onto the direction of the gradient;

$Q_{n 0}$ - initial momentum of movement of the material in a projection onto the direction of the gradient $Q_{n 0}=0$ as the material was at rest.

Force $\vec{N}_{P}$ acts as the force of static pressure with the assumption of equality in all directions, similar to Pascal's law for liquids, yet on condition that there is no pressure from the rear side of the helical surface:

$$
N_{P}=\gamma \cdot g\left(\rho_{\kappa 1}-D-\rho \cdot \sin \varphi\right) d S,
$$

where $\rho_{k 1}$ - maximum radius of the external auger of the mixer,

$D$ - vertical height of an unfilled mixer volume $D=\rho_{k 1}\left(1-\sin \varphi_{0}\right)$;

$\gamma$ - bulk density of the material;

$g$ - acceleration of gravity;

$l$ - height of the overlying layers above the elementary area (Fig. 3);

$y$ - vertical Cartesian coordinate measured from the axis of the auger.

Fig. 3 depicts the filling degree of the mixer and the thickness of the material layer $l$ to the location of the elementary helical surface $d S$.

The total friction force arising from the transfer of the material along axis $z$ is determined according to the formula:

$$
\sum F_{C}=\iint_{(S)} g \cdot \gamma \cdot \cos (\operatorname{grad}(f) \vec{k}) \cdot h \cdot k_{c} d S
$$


We will accept the following assumption that the friction force considered is proportional to the height of the material layer along axis $y$. We denote the depth of the layer by $l$ :

$$
l=\left(\rho_{\kappa 1}-D-y\right) \text {. }
$$

Then the average depth of the material layer $l_{c p}$ is determined from the expression:

$$
l_{c p}=\frac{\int_{\varphi_{0}}^{\varphi_{k}}\left(\int_{\rho_{0}}^{\rho_{\kappa}}\left(\rho_{\kappa 1}-D-\rho \cdot \sin \varphi\right) \sqrt{a^{2}+\rho^{2}} d \rho\right) d \varphi}{\int_{\varphi_{0}}^{\varphi_{k}}\left(\int_{\rho_{0}}^{\rho_{k}} \sqrt{a^{2}+\rho^{2}} d \rho\right) d \varphi} .
$$

Let us determine the relationship between the forces $\vec{F}_{C}$ and $\vec{N}_{C}$, based on the condition of uniform movement of the material along axis $z$ :

$$
N_{C}=g \cdot \gamma \cdot h \cdot k_{c} \frac{\left(\rho_{\kappa 1}-D-\rho \cdot \sin \varphi\right)}{l_{c p}} d S .
$$

During the interaction of the material with the auger the material acquires the velocity $\vec{v}$, composed of the transfer $\vec{v}_{e}$ (having a projection onto the gradient) and relative components $\vec{v}_{r}$ (having no projection onto the gradient), then

$$
Q_{n}=-\gamma \cdot v_{e}^{2} \cdot t \cdot d S \frac{\left(\frac{a}{\rho}\right)^{2}}{\left(\frac{a}{\rho}\right)^{2}+1}
$$

After substitution of the corresponding values into the theorem about the change in the momentum of movement (12), we write:

$$
-\gamma \cdot v_{e}^{2} \cdot t \cdot d S \frac{\left(\frac{a}{\rho}\right)^{2}}{\left(\frac{a}{\rho}\right)^{2}+1}-0=-N_{B} \cdot t+N_{P} \cdot t+N_{C} \cdot t
$$

From where, taking into account (13), we have the correlation for the force $N_{M}$ :

$$
N_{M}=N_{B}=\gamma \cdot v_{e}{ }^{2} \cdot d S \frac{\left(\frac{a}{\rho}\right)^{2}}{\left(\frac{a}{\rho}\right)^{2}+1}+\gamma \cdot g\left(\rho_{k 1}-D-\rho \sin \varphi\right) d S+g \cdot \gamma \cdot h \cdot k_{c} \frac{\left(\rho_{\kappa 1}-D-\rho \cdot \sin \varphi\right)}{l_{c p}} \cdot d S
$$

In expression (20), we divide the factor $\lambda$ into components:

$$
\begin{gathered}
\gamma \cdot v_{e}^{2} \cdot d S \frac{\left(\frac{a}{\rho}\right)^{2}}{\left(\frac{a}{\rho}\right)^{2}+1} ; \\
\lambda_{Q}=\frac{|\operatorname{grad}(f)|}{\lambda_{p}}=\frac{\gamma \cdot g\left(\rho_{\kappa 1}-D-\rho \cdot \sin \varphi\right) d S}{|\operatorname{grad}(f)|} ;
\end{gathered}
$$




$$
\lambda_{c}=\frac{\gamma \cdot h \cdot k_{c} \frac{\left(\rho_{\kappa 1}-D-\rho \sin \varphi\right)}{l_{c p}} \cdot d S}{|\operatorname{grad}(f)|} ;
$$

Using $\lambda_{Q}$ from expression (21) in (11) and substituting the result into (2), we find the torque obtained by acting upon the material at a preset angular velocity $\omega$ :

$$
M_{V R Q}=\int_{\varphi_{0}}^{\varphi_{k}}\left(\int_{\rho_{0}}^{\rho_{K}} \gamma \cdot \omega^{2} \cdot \frac{a^{2}\left(a+k_{T R} \rho\right)}{\left(a^{2}+\rho^{2}\right)} \rho^{3} d \rho\right) d \varphi .
$$

In a similar way, on the basis of the second term in expression (20), we write:

$$
M_{V R P}=\int_{\varphi_{0}}^{\varphi_{k}}\left(\int_{\rho_{0}}^{\rho_{K}}\left(a+k_{T R} \rho\right) \frac{\gamma \cdot g\left(\rho_{\kappa 1}-D-\rho \cdot \sin \varphi\right)}{\sqrt{\left(\frac{a}{\rho}\right)^{2}+1}} \sqrt{a^{2}+\rho^{2}} d \rho\right) d \varphi
$$

We also determine the contribution to the torque of the third term in expression (20):

$$
M_{V R C}=\int_{\varphi_{0}}^{\varphi_{k}}\left(\int_{\rho_{0}}^{\rho_{\kappa}}\left(a+k_{T R} \rho\right) \frac{g \cdot \gamma \cdot h \cdot k_{c} \frac{\left(\rho_{k 1}-D-\rho \cdot \sin \varphi\right)}{l_{c p}}}{\sqrt{\left(\frac{a}{\rho}\right)^{2}+1}} \sqrt{a^{2}+\rho^{2}} d \rho\right) d \varphi
$$

Consequently, the torque is equal to the sum of the three components

$$
M_{V R}=M_{V R Q}+M_{V R P}+M_{V R C} .
$$

The power consumed by the engine is determined from the expression:

$$
P=\omega \cdot M_{V R} \text {. }
$$

\section{Results and discussion}

The obtained expressions made it possible to carry out theoretical studies of the dependence of the value of power consumed by the engine for the drive of the working tools of the mixer upon structural and technological factors. Based on the calculation results, two-dimensional sections have been constructed. The direction of the lines in the presented figures and their form of the change are the same for the internal, medium and external augers; therefore, the figures show two-dimensional sections only for the medium augur of the mixer, but the results are described for all three augers.

Fig.4 presents the dependence of the power value upon the rotational speed of the mixer shaft and the density of the material for an average screw: a) a two-dimensional cross section, constructed according to the results of the calculation for the first component; b) a two-dimensional cross section, constructed according to the calculation results for the second component; c) a two-dimensional cross section, constructed according to the results of the calculation for the third component; d) a twodimensional cross section, representing a change in the total value of power in all three components. Analysis of Fig. 4a shows that a two-dimensional cross section, constructed in accordance with the results of the calculation according to the first component, has a curvilinear dependence. 
a)

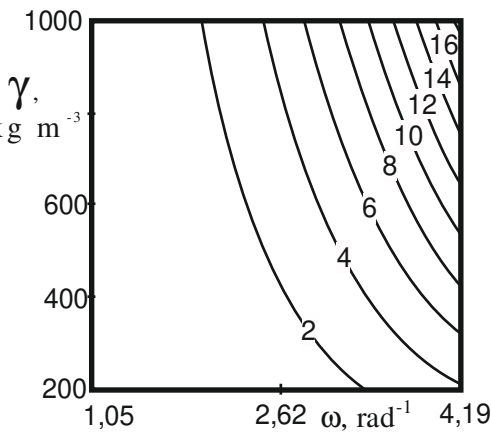

c)

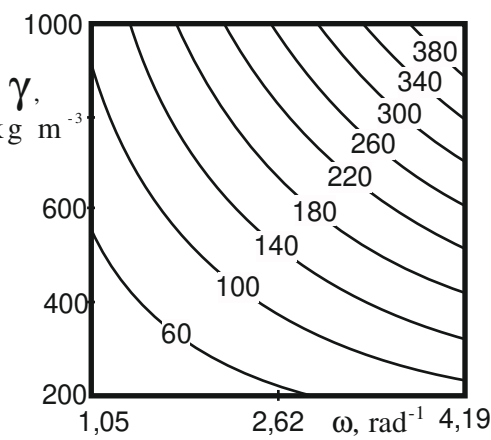

b)

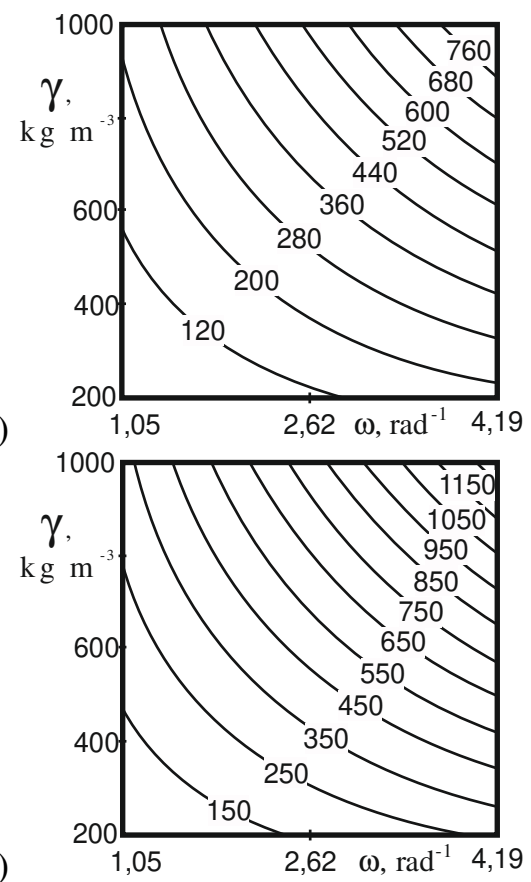

Fig. 4. Two-dimensional sections of the response surfaces, constructed by: a - the first term;

$\mathrm{b}$ - the second term; $\mathrm{c}$ - the third term; $\mathrm{d}$ - the total value of power for all three summands

When the rotation frequency of the mixer shaft changes from 10 to $40 \mathrm{~min}^{-1}\left(1.05\right.$ to $\left.4.19 \mathrm{rad} \cdot \mathrm{s}^{-1}\right)$ and the density of the material is from 200 to $1000 \mathrm{kgm}^{-3}$, the value of power changes from $0.06 \mathrm{~W}$ to $18.5 \mathrm{~W}$ for the medium auger, from $0.07 \mathrm{~W}$ to $21 \mathrm{~W}$ for the external auger and from 0.015 to $4.7 \mathrm{~W}$ for the internal auger. The two-dimensional cross section (Fig. 4b), constructed according to the second component, has a curvilinear dependence. When the rotation frequency of the mixer shaft changes from 1.05 to $4.19 \mathrm{rad} \cdot \mathrm{s}^{-1}$ and the material density varies from 200 to $1000 \mathrm{~kg} \cdot \mathrm{m}^{-3}$, the value of power changes from $40 \mathrm{~W}$ to $850 \mathrm{~W}$ for the medium auger, from $100 \mathrm{~W}$ to $2100 \mathrm{~W}$ for the external auger, and from 15 to $380 \mathrm{~W}$ for the internal auger. The two-dimensional cross section (Fig. 4c), constructed according to the third component, has a curvilinear dependence. When the rotation frequency of the mixer shaft changes from 1.05 to $4.19 \mathrm{rad} \cdot \mathrm{s}^{-1}$ and the material density varies from 200 to $1000 \mathrm{~kg} \cdot \mathrm{m}^{-3}$, the value of power changes from $20 \mathrm{~W}$ to $420 \mathrm{~W}$ for the medium auger, from 30 $\mathrm{W}$ to $630 \mathrm{~W}$ for the external auger, and from 5 to $180 \mathrm{~W}$ for the internal auger. The two-dimensional cross section (Fig. 4d), constructed as a result of the sum of all three components, has a curvilinear dependence. When the rotation frequency of the mixer shaft changes from 1.05 to $4.19 \mathrm{rad} \cdot \mathrm{s}^{-1}$ and the material density varies from 200 to $1000 \mathrm{~kg} \cdot \mathrm{m}^{-3}$, the value of power changes from $60.06 \mathrm{~W}$ to $1288.5 \mathrm{~W}$ for the medium auger, from $130.07 \mathrm{~W}$ up to $2751 \mathrm{~W}$ for the external auger, and from 20.015 to $564.7 \mathrm{~W}$ for the internal auger. Analysis of Fig. 4a allows drawing the following conclusions. A change in the rotation frequency of the shaft from 1.05 to $4.19 \mathrm{rad} \mathrm{s}^{-1}$ leads to an increase in power from 0.07 to $3.6 \mathrm{~W}$ at a density of $200 \mathrm{~kg} \cdot \mathrm{m}^{-3}$, and a change in frequency from 1.05 to 4 . $\mathrm{rad} \cdot \mathrm{s}^{-1}$ increases the value of power from 0.07 to $18.5 \mathrm{~W}$ with the material density of $1000 \mathrm{~kg} \cdot \mathrm{m}^{-3}$. Examination of the surfaces, constructed according to other components, allows to say that a change in the value of the rotation frequency and density of the material has the same impact upon the nature of the change in the value of power. Analysis of the two-dimensional cross sections, presented in Fig. 4, allows a conclusion that the rotation frequency of the working tools of the mixer should not exceed $2.62 \mathrm{rad} \cdot \mathrm{s}^{-1}$, and the components of the mixture should not be heavily disintegrated before mixing. Fig. 5 presents the dependence of the power value upon the rotational speed of the mixer shaft and the pitch of the screw tape for the average screw: a) a two-dimensional cross section, constructed according to the results of calculation for the first component; b) a two-dimensional cross section, constructed according to the calculation results for the second component; c) a two-dimensional cross section, constructed according to the results of the calculation for the third component; d) a twodimensional cross section, representing a change in the total value of power for all three components. 
a)

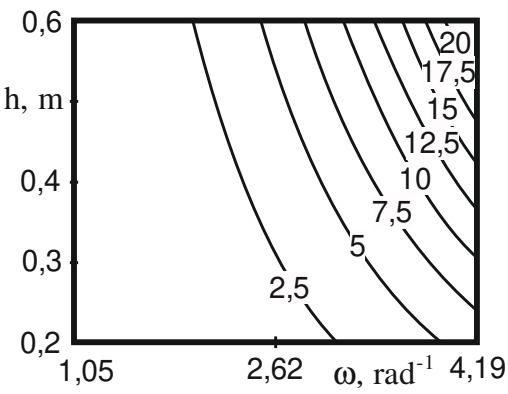

c)

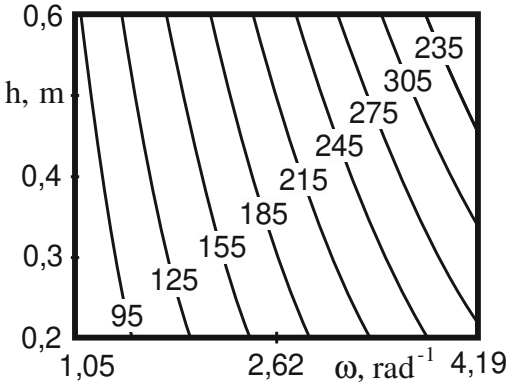

b)

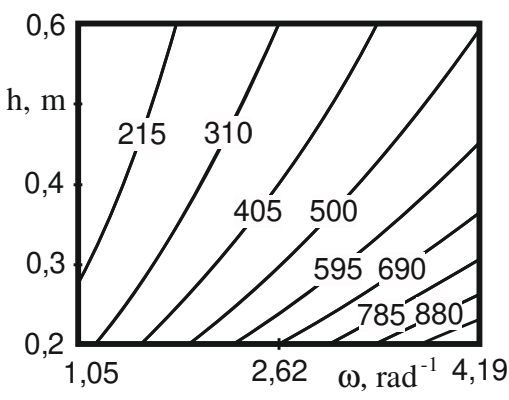

d)

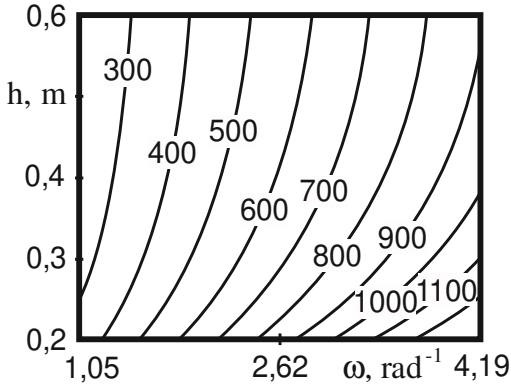

Fig. 5. Two-dimensional sections of the response surfaces, constructed according to: $a-$ the first term; $\mathrm{b}$ - the second term; $\mathrm{c}$ - the third term; $\mathrm{d}$ - the total power for all three summands

Analysis of Fig. 5a shows that the two-dimensional cross section, constructed according to the calculation results performed according to the first component, has a curvilinear dependence. When the rotation frequency of the mixer shaft changes from 1.05 to $4.19 \mathrm{rad} \cdot \mathrm{s}^{-1}$ and the pitch of the auger turn is from 0.2 to $0.6 \mathrm{~m}$, the value of power changes from $0.09 \mathrm{~W}$ to $22.7 \mathrm{~W}$ for the medium auger, from $0.16 \mathrm{~W}$ to $29 \mathrm{~W}$ for the external auger, and from 0.04 to $9.5 \mathrm{~W}$ for the internal auger. The twodimensional cross section (Fig. 5b), constructed according to the second component, has a curvilinear dependence.

When the rotation frequency of the mixer shaft changes from 1.05 to $4.19 \mathrm{rad} \cdot \mathrm{s}^{-1}$ and the auger pitch is from 0.2 to $0.6 \mathrm{~m}$, the value of power changes from $120 \mathrm{~W}$ to $1070 \mathrm{~W}$ for the medium auger, from $250 \mathrm{~W}$ to $4050 \mathrm{~W}$ for external auger, and from 48 to $1200 \mathrm{~W}$ for the internal auger. The twodimensional cross section (Fig. 5c), constructed according to the third component, indicates that, when the rotation frequency of the mixer shaft changes from 1.05 to $4.19 \mathrm{rad} \cdot \mathrm{s}^{-1}$ and the pitch of the auger turn is from 0.2 to $0.6 \mathrm{~m}$, the value of power varies from $60 \mathrm{~W}$ to $370 \mathrm{~W}$ for the medium auger, from $100 \mathrm{~W}$ to $525 \mathrm{~W}$ for the external auger, and from 20 to $200 \mathrm{~W}$ for the internal auger.

Analysis of the two-dimensional cross section (Fig. 5d), constructed as a result of the sum of all three components, reveals that, when the rotation frequency of the mixer shaft changes from 1.05 to $4.19 \mathrm{rad} \cdot \mathrm{s}^{-1}$ and the pitch of the auger turn is from 0.2 to $0.6 \mathrm{~m}$, the value of power varies from $180.09 \mathrm{~W}$ to $1462.7 \mathrm{~W}$ for an medium screw, from $350.16 \mathrm{~W}$ to $4604 \mathrm{~W}$ for an external auger, and from 68.04 to $1409.5 \mathrm{~W}$ for an internal auger. By analysing Fig. 5, we can draw the following conclusion. The frequency of rotation of the working tools of the mixer to reduce energy consumption should not exceed $2.09 \mathrm{rad} \cdot \mathrm{s}^{-1}$, and the pitch of the medium auger should be within the range from 0.4 to $0.6 \mathrm{~m}$. Fig. 6 presents the dependence of the power value upon the height of the auger belt and its pitch for the average auger: a) a two-dimensional cross section, constructed according to the results of calculation for the first component; b) a two-dimensional cross section, constructed according to the calculation results for the second component; c) a two-dimensional cross section, constructed according to the results of calculation for the third component; d) a two-dimensional cross section, representing a change in the total value of power over all three components.

The two-dimensional cross section (Fig. 6a), constructed according to the first component, has a curvilinear dependence. When changing the width of the auger belt from 30 to $120 \mathrm{~mm}$ and the pitch of the auger turn from 0.2 to $0.6 \mathrm{~m}$, the value of power changes from $0.55 \mathrm{~W}$ to $7.1 \mathrm{~W}$ for the medium auger, from $0.6 \mathrm{~W}$ to $9.5 \mathrm{~W}$ for the external auger, and from 0.1 to $2.0 \mathrm{~W}$ for the internal auger. The greater is the pitch, the greater is power. When the pitch of the auger turn is increased from 0.2 to $0.6 \mathrm{~m}$ with the width of the auger belt being $120 \mathrm{~mm}$, the value of power increases from 1.9 to $7.1 \mathrm{~W}$ for the medium auger, from 1.5 to $9.5 \mathrm{~W}$ for the external auger, and from 0.1 to $1.9 \mathrm{~W}$ for the internal 
auger. The two-dimensional cross section (Fig. 6b), constructed according to the second component, has a curvilinear dependence. When changing the width of the auger belt from 30 to $120 \mathrm{~mm}$ and the pitch of the auger turn from 0.6 to $0.2 \mathrm{~m}$, the value of power changes from $165 \mathrm{~W}$ to $1115 \mathrm{~W}$ for the medium auger, from $350 \mathrm{~W}$ to $4350 \mathrm{~W}$ for an external auger, and from 50 to $770 \mathrm{~W}$ for the internal auger. This is determined by the fact that the basis of calculation according to the second component is friction of the material across the surface of the auger.

a)

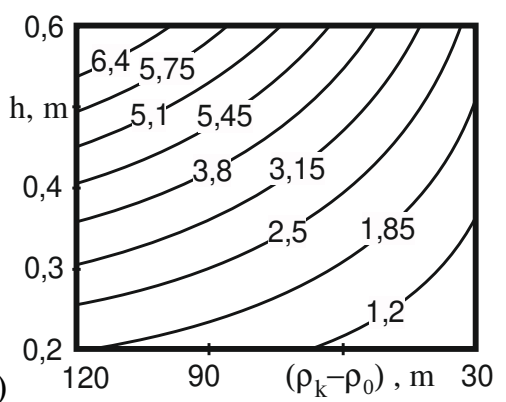

c)

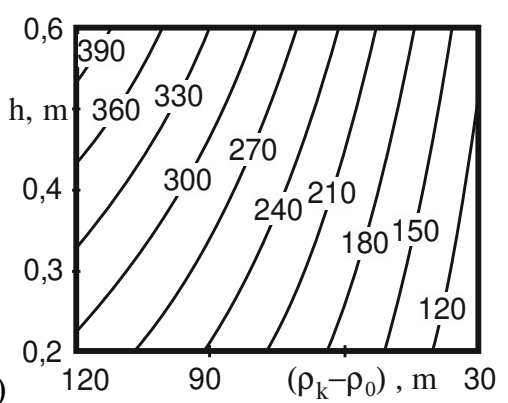

b)

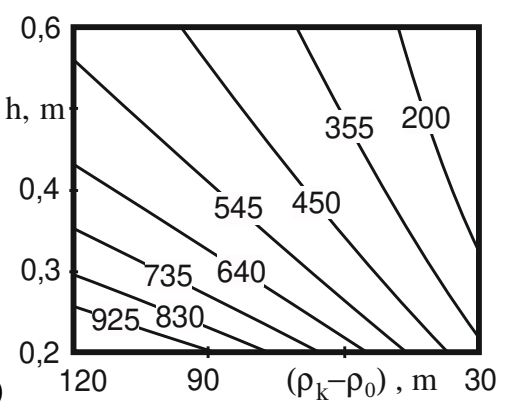

d)

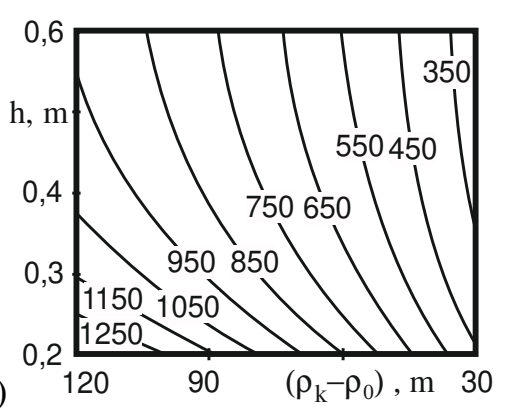

Fig. 6. Two-dimensional sections of the response surfaces, constructed according to: $a-$ the first term; $\mathrm{b}$ - the second term; $\mathrm{c}$ - the third term; $\mathrm{d}$ - the total power for all three summands

Consequently, when decreasing the pitch of the auger, the number of the turns of the auger, accordingly, the contact area of the material with the belt of the auger increases. The two-dimensional cross section (Fig. 6c), constructed according to the third component, has a curvilinear dependence. When changing the width of the auger belt from 30 to $120 \mathrm{~mm}$ and the pitch of the auger turn from 0.2 to $0.6 \mathrm{~m}$, the value of power changes from $90 \mathrm{~W}$ to $400 \mathrm{~W}$ for the medium auger, from $135 \mathrm{~W}$ to $600 \mathrm{~W}$ for the external auger, and from 25 to $150 \mathrm{~W}$ for the internal auger.

The two-dimensional cross section (Fig. 6d), constructed as a result of the sum of all the three components, has a curvilinear dependence. When changing the width of the auger belt from 30 to $120 \mathrm{~mm}$ and the pitch of the auger turn from 0.6 to $0.2 \mathrm{~m}$, the value of power changes from $300 \mathrm{~W}$ to $1380 \mathrm{~W}$ for the medium auger, from $700 \mathrm{~W}$ to $4800 \mathrm{~W}$ for the external auger, and from 110 to $905 \mathrm{~W}$ for the internal auger. Since the calculation results of power are most of all affected by the component found by the second calculation method, the maximum power is achieved with a belt of $120 \mathrm{~mm}$ and a pitch of $200 \mathrm{~mm}$.

Analysis of the two-dimensional cross-sections, shown in Fig.6, allows drawing a conclusion that, in order to achieve the least power consumption to drive the mixer working tools, the height of the auger belt should be from 30 to $50 \mathrm{~mm}$, and the pitch of the medium auger belt - from 0.4 to $0.6 \mathrm{~m}$. Experimental studies have been carried out for the structural and technological parameters determined as a result of theoretical studies. Based on the obtained results, dependencies have been constructed (Fig. 7). The results of theoretical and experimental studies, presented in Fig.7 of dependences 1 and 2 , indicate that the discrepancies between the theoretical and the experimental values are insignificant. The coefficient of variation, when loading the hopper of the mixer to $75 \%$, is $6.3 \%$.

Based on the quality of mixing the material, the recommended filling of the mixer and the efficient use of the mixing chamber, the optimal loading of the mixer is $55-75 \%$ [6]. 


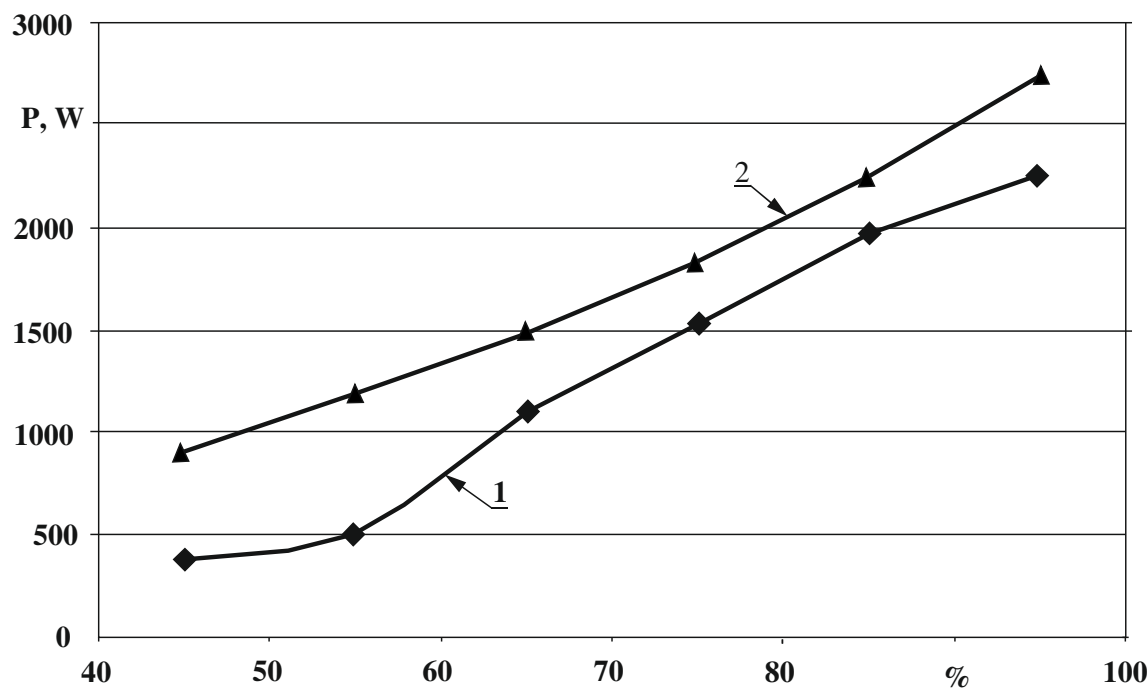

Fig. 7. Dependence on the volume of the hopper filling: 1 - power consumption during the experimental studies; 2 - the power obtained by calculation

\section{Conclusions}

Analysis of the impact of the rotation frequency of the mixer shaft and the material density upon the power consumed for the drive can witness that the rotation frequency of the mixer working tools should not exceed $2.62 \mathrm{rad} \cdot \mathrm{s}^{-1}$, and, before mixing, the components of the mixture should not be heavily disintegrated.

Changing the rotation frequency of the mixer shaft and the pitch of the auger turn, depending on the value of the consumed power, allows to say that the rotation frequency of the mixer working tools in order to reduce energy consumption should not exceed 2.09 rad.s $^{-1}$, and the pitch of the medium auger should be within the range of 0.4 up to $0.6 \mathrm{~m}$.

Investigations of the dependence of power consumption upon the height of the auger belt and its pitch allow a conclusion that in order to achieve minimum power consumption to drive the mixer working tools, the height of the auger belt should be from 30 to $50 \mathrm{~mm}$, and the pitch of the medium auger belt should be $0.4-0.6 \mathrm{~m}$.

The results of the theoretical and experimental studies indicate that the discrepancies between the theoretical and the experimental values are insignificant. The coefficient of variation, when loading the hopper of the mixer to $75 \%$, is $6.3 \%$.

\section{References}

[1] Heinrichs A.J., Buckmaster D.R., Lammers B.P. Processing, mixing, and particle size reduction of forages for dairy cattle.Journal of Animal Science, Volume 77, Issue 1, 1999, pp. 180-186.

[2] СкоркинВ. Современные требования производства конкурентоспособной молочной продукции (Contemporary requirements for the production of competitive dairy products). Вестник ВНИИМЖ (Vestnik VNIIMZh), No 4 (28), 2017. pp. 4-9. (In Russian)

[3] Sysuev V., Ivanovs S., Savinyh P., Kazakov V. Movement and transformation of grain in twostage crusher. Engineering for Rural Development, Proceedings, Vol.14, 2015, pp. 22-27;

[4] MarczukA., CabanJ., SavinykhP., TurubanovN., ZyryanovD. Maintenance research of a horizontal ribbon mixer. Eksploatacja I Niezawodnosc - Maintenance and Reliability, 2017, vol. 19 (1). pp. 121-125.

[5] Heinrichs A.J., Buckmaster D.R., Hevko R.B., Liubin M.V., Tokarchuk O.A.,Lyashuk O.L., Pohrishchuk B.V.,Klendii O.M. Determination of the parameters of transporting and mixing feed mixtures along the curvilinear paths of tubular conveyors. INMATEH - Agricultural Engineering, Volume 55, Issue 2, 2018. pp. 97-104.

[6] McCoy R.A., Behnke K.C., Hancock, J.D., McEllhiney R.R. Effect of mixing uniformity on broiler chick performance. Poultry Science, 73, 1994. pp. 443-451. 
[7] Нис Я.З. Физико-механические, физико-химические и теплофизические свойства сырья и готовых продуктов пищевой промышленности (Physico-mechanical, physico-chemical and thermophysical properties of raw materials and finished products of the food industry), Новочеркасск, 2012. 28 p. (In Russian).

[8] Fürll C., Hoffmann T. Flow properties of components for dry compound feed. Powder Technology, vol.235, 2013, pp. 838-841.

[9] Groesbeck C.N., Goodband R.D., Tokach,M.D., Dritz S.S., Nelssen J.L., DeRouchey J.M. Diet mixing time affects nursery pig performance. Journal of Animal Science, vol.85 (7), 2007, pp. 1793-1798.

[10] Сысуев B.А.Кормоприготовительные машины. Теория, разработка, эксперимент (Feed preparation machines. Theory, development, experiment), Киров, Т.2, 2009. $496 \mathrm{c.}$

[11]Bulgakov V., Pascuzzi S., Nadykto V., Ivanovs S. A mathematical model of the plane-parallel movement of an asymmetric machine-and-tractor aggregate. Agriculture, Vol. 8(10), 2018, art. 151 . 